Michele Taruffo

«RACIONALIDAD Y CRISIS
DE LA LEY PROCESAL»

DOXA 22 (1999) 


\section{RACIONALIDAD Y CRISIS DE LA LEY PROCESAL}

Michele Taruffo

Universidad de Pavía

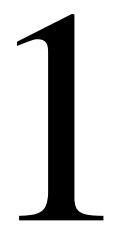

Las consideraciones que iré desarrollando atañen, sobretodo, al proceso civil, aunque quizás una buena parte de ellas podría referirse también, con pocas variaciones, al proceso penal. Naturalmente, tendré presente de modo particular la situación italiana pero, bajo varios aspectos, muchos de los problemas de los cuales tengo intención de hablar son comunes a muchos países, bien con grados diversos de intensidad, bien en diferentes modalidades.

Con la finalidad de poner un mínimo de orden en la discusión de temas tan complejos, dividiré mi exposición en cuatro puntos fundamentales. Trataré, en primer lugar, de diferenciar dos nociones distintas de «racionalidad de la ley procesal». Posteriormente, trataré de determinar en qué consiste la crisis de la ley procesal, refiriéndome a estas nociones de racionalidad. Trataré después de determinar, al menos, algunos de los motivos de estas formas de crisis. Por último, dedicaré algunas reflexiones a la posibilidad de presentar remedios que pongan fin a la crisis o al menos que reduzcan su gravedad en alguna medida.

Por razones de tiempo y de espacio, mi exposición será extremadamente sintética y esquemática. Muchas de las cosas de las que hablaré serán únicamente enunciadas o puestas de manifiesto sin el análisis que requerirían. Por ello, en muchos puntos, se correrá el riesgo de hacer afirmaciones genéricas o no justificadas adecuadamente. De todos modos, espero que el discurso en su conjunto parezca sensato.

2. El concepto de racionalidad puede relacionarse con la ley procesal en, al menos, dos sentidos principales: racionalidad como coherencia de la ley procesal y racionalidad como funcionalidad (o racionalidad instrumental) de la misma ley.

La coherencia de la ley procesal es una característica que se puede apreciar si imaginamos que se ponen todas las normas procesales de un ordena- 
miento en un mismo plano, como para componer un mosaico, y se verifica si las distintas partes de éste -sea a nivel de macroanálisis, sea a nivel de cada una de las particulares partes del mosaico- son recíprocamente coherentes. Desde esta perspectiva se pueden apreciar algunas propiedades que puede presentar la ley procesal, y se puede -hipotéticamente- establecer también en qué grado estas propiedades están presentes en un sistema procesal dado. Estas propiedades son el orden, o sea, la secuencia en la cual vienen reguladas las actividades procesales (pero también las relaciones entre normas generales y disposiciones específicas); la unidad, que deriva de tratarse de normas que forman un contexto único con un núcleo de normas fundamentales y, posiblemente, pocas normas especiales (p. ej.: un código procesal con pocas leyes o normas procedimentales situadas fuera de él); la plenitud, que se produce cuando la ley procesal regula todos los aspectos relevantes de los mecanismos procedimentales de un modo suficientemente analítico, dejando lo menos posible a la fantasía de los intérpretes; y la simplicidad, que existe cuando la ley regula de modo claro un procedimiento que se resuelve en un mecanismo relativamente no complejo. Quizás estas propiedades no agotan el concepto de racionalidad como coherencia de la ley procesal, pero tal vez puede decirse que una ley procesal que sea ordenada, unitaria, completa y simple en un nivel apreciable, es racional en una sensata acepción del término.

Se puede también decir que la ley procesal es racional en sentido instrumental si regula un procedimiento que sirve adecuadamente a los fines de la administración de justicia. Naturalmente, el juicio acerca de la racionalidad instrumental de un proceso varía en función de los fines que a éste se le asignen. Sin embargo, se puede estar de acuerdo en líneas generales en el hecho de que, si lo que se espera de un proceso son cosas como la actuación de la ley en el caso particular, la formulación de decisiones justas y la efectiva tutela de los derechos de los sujetos interesados, se puede encontrar, al menos, un punto de referencia general. Respecto a esta finalidad se podrá decir, p. ej., que es racional un proceso que se desarrolle en un tiempo razonable, que incluya normas probatorias idóneas para lograr decisiones fácticas acertadas, que comprenda instrumentos para la realización efectiva de los derechos, que incluya remedios para el control relativo a la justicia de las decisiones, etc.

Naturalmente, estas dos nociones de racionalidad de la ley procesal no son del todo contrarias entre sí. Son distintas, no se implican recíprocamente (es posible imaginar una ley coherente pero ineficiente; es, por el contrario, difícil - pero no imposible- imaginar una ley procesal instrumentalmente racional pero no coherente), pero son perfectamente compatibles. Se podría decir más bien que un sistema procesal óptimo es aquél en el cual 
la ley procesal es racional, tanto en el sentido de la coherencia como en el sentido de la funcionalidad instrumental respecto de los fines que se consideran propios de la administración de justicia.

3. La crisis que afecta a muchas legislaciones procesales es reconducible a una serie de razones. Algunas de ellas hacen referencia a la racionalidad como coherencia de la ley procesal, mientras que otras se refieren a su funcionalidad instrumental.

En relación con la crisis de coherencia, se pueden subrayar, sobre todo, tres fenómenos, que se hacen particularmente evidentes en la experiencia de muchos sistemas procesales contemporáneos. Un primer fenómeno hace referencia a la escasa posibilidad de asegurar el orden y la unidad de la ley procesal, pues prevalece la tendencia opuesta a la fragmentación y, por consiguiente, a la falta de orden sistemático. Son muchas las causas de este fenómeno, pero la principal -que merece ser subrayada- es la extendida tendencia a crear «estatutos privilegiados» de naturaleza procesal para particulares grupos o categorías de personas. Una de las consecuencias de la disfunción general del proceso (de la cual hablaré dentro de poco), y de la extrema dificultad de realizar reformas globales del derecho procesal, es la creación de procedimientos especiales (o de formas de «tutela diferenciada») que deberían ofrecer una mejor protección judicial de los derechos de determinados sujetos. El problema no es nuevo, pues hay ejemplos clásicos (basta pensar en las juridictions d'exception en Francia), pero tras la segunda Guerra Mundial las manifestaciones son cada vez más numerosas. Además del caso tradicional del procedimiento de enjuiciamiento para la tutela rápida de los acreedores (o sólo de aquellos que gozan de prueba escrita de su crédito), se puede pensar en los procesos especiales para los conflictos laborales y, más recientemente, en los procedimientos para la tutela de los consumidores. En definitiva, la unitariedad del sistema procesal se rompe cada vez que el legislador tiene intención de sustraer determinados sujetos o grupos de sujetos al funcionamiento - a menudo ineficiente- de la ley procesal «general». Esto comporta, normalmente, una mejora en la satisfacción de los derechos de estos sujetos, pero tiene efectos críticos sobre el sistema globalmente entendido. En efecto, de un lado, se crean numerosas situaciones de tratamiento dispar desde el punto de vista procesal, porque algunos sujetos (o, lo que es lo mismo, algunos derechos) son tutelados mucho mejor de lo que lo es el «normal» titular de una situación jurídica no privilegiada. De otro lado, hay presiones, por parte de grupos de sujetos, para la creación de «procesos especiales» cada vez más numerosos, cada uno referido a un grupo cualificado de sujetos, en una especie de «carrera de privilegios». El efecto global de esto es que el ordenamiento procesal asume una 
estructura «de piel de leopardo», con islas de tutela eficiente para determinados sujetos sobre un fondo de tutela ineficiente reservada para el ciudadano «común», que no pertenece a un grupo privilegiado o no es titular de una situación jurídica privilegiada.

Un segundo fenómeno de crisis es el relativo a la creciente incompletitud de la ley procesal. Frente a la siempre mayor complejidad de las relaciones sociales y económicas y, por tanto, de las situaciones jurídicas necesitadas de tutela jurisdiccional, el legislador procesal está frecuentemente atrasado en decenios respecto al surgimiento de los problemas que debe afrontar. Esto comporta un doble orden de consecuencias. Por un lado, aumenta el número de los sujetos (o de los derechos) que no vienen de hecho tutelados en vía jurisdiccional, porque faltan instrumentos procesales ad hoc (valga por todos el ejemplo de los intereses colectivos o supraindividuales) o porque existen lagunas y disfunciones tales que impiden de hecho el acceso a la tutela jurisdiccional (valga el ejemplo de la falta o ineficiencia de las normas para la defensa en juicio de los no adinerados). La ley procesal tiende, pues, a hacerse cada vez más incompleta, sea sobre el plano formal (o sea: por la falta de normas que serían necesarias), sea sobre el plano sustancial (o sea: por la falta de funcionamiento de normas que ya existen formalmente).

Un tercer fenómeno de crisis hace referencia a la creciente complejidad y confusión de la ley procesal. La legislación procesal es cada vez peor y se realiza de forma más apresurada. Muy frecuentemente, ésta emana del impulso de exigencias eventuales, o del intento de encontrar remedios rápidos a cada uno de los problemas de disfuncionalidad del sistema. Además, normalmente se introducen reformas parciales inciertas, inestables, porque están sujetas a rápidos cambios de pensamiento del legislador, confeccionadas al margen de todo diseño orgánico y sistemático de reforma del proceso. Las tentativas de reforma del proceso civil realizadas en Italia entre 1990 y 1995, no terminadas todavía, y ya claramente fallidas en gran medida, son un buen ejemplo de este fenómeno. Otros países han sido más afortunados (me refiero a las reformas francesa y alemana en torno a la mitad de los años 70), o tratan de invertir esta tendencia (pienso en el Anteproyecto español de un nuevo código de procedimiento civil, que está actualmente en discusión). Sin embargo, permanece muy difundida la tendencia a elaborar normativas procesales muy complejas y diferenciadas, mientras se hace cada vez más difícil la individualización de modelos de proceso que sean bastante simples y bien definibles. Además, a menudo se tiene la impresión de una creciente confusión derivada de la dificultad de interpretar correctamente normas cada vez más vagas, fragmentarias y técnicamente defectuosas, de la casi total imposibilidad de distinguir principios y reglas específi- 
cas y de la frecuente presencia, en el mismo conjunto de normas, de orientaciones y finalidades opuestas.

No menos variadas y complejas son las manifestaciones de la crisis de funcionalidad de la ley procesal. Sobre todo, está muy generalizada (quizás con la única excepción de Alemania) la crisis de efectividad de la tutela jurisdiccional, que deriva esencialmente de los retrasos cada vez más largos de la justicia, frente a la creciente necesidad de soluciones rápidas y eficaces de las controversias. El dicho justice delayed is justice denied, es cada vez más aplicable a muchos ordenamientos: los períodos largos, y a veces absurdos, de la justicia representan en gran medida el principal factor de crisis del sistema procesal. La efectividad del proceso está en crisis también en aquellos países, como Gran Bretaña, que tradicionalmente eran señalados como modelos de rapidez y funcionalidad. Esto conlleva varias consecuencias relevantes. De un lado, se hacen cada vez más numerosas las áreas en las cuales, de hecho, no se administra realmente una justicia aceptable. Así, la elección más racional para el titular de un derecho consiste en no hacer valer este derecho en juicio, porque el tiempo (y, por tanto, también los costes) necesarios para obtener una sentencia definitiva serían excesivos. Por lo tanto, se produce una sustancial elusión de la necesidad de tutela procesal y se vuelven ineficaces las garantías de acceso a los tribunales que están recogidas en numerosas Constituciones contemporáneas. Pero la situación no es mejor para quien no se rinde desde el principio y va a juicio: varios años para obtener una sentencia en primera instancia, más otros años para el juicio de apelación, más otros años todavía para el juicio de casación y eventualmente algún año más para el juicio de reenvío, representan una experiencia desastrosa y costosa, en la cual, quien resulta realmente beneficiado es el deudor incumplidor que se lucra de las disfunciones de la justicia. Un aspecto muy importante de esta situación es que los inconvenientes y las disfunciones del sistema procesal no tienen -como es obvio- las mismas consecuencias para todos. Por un lado, como se ha señalado, hay sujetos o categorías de sujetos que disfrutan de formas de tutela privilegiadas frente a otros. Por otro lado, el inconveniente representado por los retrasos y los costes del proceso funciona de un modo muy diverso según el tipo de sujetos: el sujeto social y económicamente «débil» sufre en mayor medida las disfunciones del sistema, y será inducido más fácilmente a no tutelar sus derechos o a abandonar un proceso demasiado largo e ineficiente. No así el sujeto social y económicamente «fuerte» (como las «partes habituales» del proceso civil: aseguradoras, organizaciones de ventas, grandes fabricantes, bancos, etc.) que tienen la posibilidad de hacer valer sus derechos sin problemas (incluso con operaciones de «política judicial» previstas expresamente), o de resistir el juicio hasta el final si son deudores (sobre todo si el acreedor es «débil»). Surgen, pues, nu- 
merosas situaciones de desigualdad sustancial, y por tanto de evidente injusticia, en el uso de los instrumentos procesales de tutela de los derechos.

Aquí hay, además, otro aspecto de la crisis de efectividad del sistema procesal que rápidamente va adquiriendo (sobre el ejemplo de Estados Unidos, pero con una fuerte difusión en Europa) una gran importancia. Se trata del fenómeno que ya se conoce comúnmente con el nombre de ADR (=Alternative Dispute Resolution), y que incluye, además de la figura tradicional del arbitraje, una serie de instrumentos de mediación y conciliación. En sustancia, la crisis de funcionalidad de la administración ordinaria de justicia se convierte en un impulso muy fuerte para la búsqueda de formas «alternativas» de solución de controversias. El fenómeno es tan evidente, y tan grave, que en muchos casos (como en Italia, aunque la tendencia está muy difundida) es el propio legislador estatal quien prevé estas alternativas, con la esperanza de que muchas controversias se resuelvan «fuera» del proceso, y por lo tanto que se reduzcan en alguna medida las dimensiones de la crisis de funcionalidad de la justicia ordinaria. Se trata de una tendencia en fase de «expansión» en varios ordenamientos, y ya tan compleja que no es posible discutirla aquí ni siquiera de modo sumario. Me limito por tanto a señalar dos puntos que considero muy importantes: a) contrariamente a lo que muchos piensan, el creciente uso de formas de ADR no es un factor positivo, sino la señal evidente de la crisis de la justicia ordinaria: es un signo de patología, no de salud del sistema procesal; b) también contrariamente a lo que muchos piensan, no todo es bueno en el ámbito de los ADR. Se vienen señalando desde hace tiempo inconvenientes, injusticias, degeneraciones y desigualdades en el funcionamiento de muchos instrumentos «alternativos», así que sería absurdo depositar en ellos una confianza indiscriminada. Al contrario, el problema actual en este sector consiste precisamente en determinar, y posiblemente eliminar, los defectos que en muchos casos se manifiestan. En resumen, probablemente es imposible -ahora, y aún más en el futuro- prescindir de instrumentos de ADR. Sin embargo, no hay que olvidar que éstos representan, de todos modos, una solución «de reserva», si no de «serie $\mathrm{B} »$, respecto de la tutela jurisdiccional de los derechos, y que no es raro que produzcan «malas»o injustas resoluciones de las controversias.

4. Siendo muy variadas y complejas las modalidades de crisis de la ley procesal, no es posible abordar en pocas líneas las razones de esta crisis, que son, a su vez, variadas y complejas. Se puede, sin embargo, tratar de determinar-obviamente en términos muy generales- los factores principales que de algún modo la explican.

Un primer factor importantísimo, al cual ya se ha hecho referencia, está representado por la violenta aceleración de las transformaciones socio-eco- 
nómicas que se verifica desde hace ya algún decenio en todo el mundo, pero especialmente en las áreas más avanzadas. Este fenómeno produce importantes problemas en muchas áreas del derecho (desde el derecho contractual al derecho tributario), pero estos problemas son especialmente graves en el ámbito de la ley procesal. El legislador procesal se encuentra con mayores dificultades para reaccionar tempestivamente ante estas transformaciones, sea por razones institucionales (cada reforma del proceso afecta a los derechos fundamentales del ciudadano, a la función del juez, a la intervención del Estado en las controversias privadas, etc.), sea por razones prácticas (esencialmente derivadas del hecho de que es muy difícil garantizar un buen funcionamiento de la justicia en condiciones de estabilidad socio-económica, y que esto se hace tendencialmente imposible en situaciones de rápida transformación). Estas dificultades se traducen en retrasos, lagunas, confusión, bajo nivel cualitativo de las normas y ausencia de un diseño orgánico en la base de la disciplina legal del proceso.

Un segundo factor muy importante es el representado por la creciente influencia (y algunas veces por su prevalencia sobre otros factores) de estímulos «corporativos» de diversa naturaleza. Como ya se ha señalado, sucede cada vez con más frecuencia que las decisiones de política procesal vienen determinadas por intereses de categorías o de grupos de sujetos: se trata, algunas veces, de los trabajadores dependientes, que obtienen un «proceso especial de trabajo» más rápido y eficaz; otras veces de los comerciantes, que mantienen tribunales propios (como los tribunaux de commerce franceses) o imponen reformas del arbitraje comercial que lo hacen más eficaz y ventajoso; de los consumidores, que solicitan u obtienen formas de tutela jurisdiccional colectiva, etc. Pero, con frecuencia, se trata de los intereses corporativos de las profesiones que operan dentro de la administración de justicia: a veces, reformas potencialmente eficaces del proceso son impedidas por la oposición de estos profesionales, extremadamente atentos a evitar cualquier innovación que pueda incidir de algún modo sobre los intereses de los abogados (en Italia se han producido numerosos y significativos ejemplos de ello); además, no es raro que la magistratura favorezca o se oponga a reformas del proceso civil o penal, a veces en nombre de los valores generales relativos al buen funcionamiento de la justicia, pero también frecuentemente para la tutela de los intereses específicos de los jueces.

Una tercera razón de la crisis está determinada por la falta de certeza que se manifiesta en torno a los fines fundamentales de la administración de justicia, y que se refleja «hacia atrás» en el modo de concebir y configurar la ley procesal, provocando conflictos y confusiones. Por ejemplo, se concibe de manera diversa la racionalidad interna y funcional de una determinada 
disciplina del proceso penal, y se configuran de formas diversas eventuales reformas, según se considere que la finalidad del proceso penal sea la de llegar a la verificación y a la punición de los delitos o, en su lugar, se opine que el proceso sirve sobre todo al imputado para hacer valer sus defensas. En el ámbito del proceso civil, el modo en que se concibe la función y la estructura del modelo procesal (por ejemplo, a propósito de la distribución de los poderes entre el juez y las partes) varía profundamente según se defina la función del proceso en términos de actuación del derecho sustancial en el caso concreto o en términos de pura y simple resolución de controversias privadas entre sujetos privados. La falta de certeza sobre la definición de los fines del proceso determina la crisis de la ley procesal en varios niveles. Por un lado, puede suceder que un modelo de proceso sea adecuado al fin A pero no al fin $\mathrm{B}$, por lo que la elección entre una y otra regulación del proceso se hace imposible -o casual y, de todos modos, difícil-incluso en el plano de eventuales reformas. Por otro lado, se hace imposible establecer si un sistema procesal dado es o no instrumentalmente funcional (y, por lo tanto, si merece o no ser cambiado), ya que una valoración de este tipo presupone, al menos, un mínimo de acuerdo en torno a los resultados que con ese instrumento se querían conseguir.

En el trasfondo de estos problemas está presente el hecho de que muchos ordenamientos se encuentran en una fase de transición socio-cultural más que de transformación económica global. En esta transición, muchos principios y valores fundamentales cambian de significado y vienen a configurarse en términos más vagos y, a menudo, conflictivos. Así, se pueden señalar importantes aspectos de la crisis de la cultura jurídica en general, que en este momento resultan capaces de construir (o reconstruir) modelos conceptuales coherentes que puedan ser usados como esquemas de referencia para nuevas leyes procesales racionales. Por ejemplo, algunos discuten si la «justicia del Estado» está siempre privilegiada respecto de las diversas formas de «justicia privada». Esto es, se pone en duda si el proceso debe concebirse todavía como un instrumento de justicia social y de tutela y realización de los derechos (sobre todo de aquéllos pertenecientes a los sujetos «débiles») o si, por el contrario, debe ser considerado como una forma regulada de combate o competición en la cual es justo y racional que venza el más fuerte. Como consecuencia de esto, también está en discusión la función institucional del juez, debiéndose decidir acerca de si es o debe ser árbitro del combate o manager, aplicador de la ley o mediador, pasivo o activo, etc. En otras ocasiones, se discuten muchos problemas que hacen referencia a la estructura del proceso, estando abierta la elección entre modelos de diversa estructura, entre formas acusatorias y dispositivas y formas inquisitivas, entre procedimientos ordinarios y procedimientos sumarios. Que 
surja esta variedad de temas de discusión, de opiniones y de contrastes es normal en una situación en la que los problemas que se refieren a la ley procesal se hacen cada día más importantes y complicados. Lo que aquí interesa poner de manifiesto es, sin embargo, la situación de gran dificultad en la que la cultura jurídica en general, y la del proceso en particular, se encuentra en este momento.

5. Frente al panorama trazado hasta ahora, puede parecer ingenuo pensar en los posibles remedios, siendo tantas y tales las razones de la crisis de la ley procesal. Pero no parece imposible trazar las líneas a lo largo de las cuales podría desarrollarse una reflexión orientada, bien a recuperar, bien a construir en términos nuevos y originales respecto al pasado, la racionalidad del proceso en una medida significativa, en los dos significados que se han distinguido al principio. Naturalmente, no es posible proponer aquí soluciones específicas a los infinitos problemas que originan la crisis de la ley procesal en los distintos sistemas.

Pero sí se pueden indicar tres direcciones de estudio y de reflexión que podrían ser útiles, al menos, para afrontar esta crisis. Ante todo, es necesario una decidida mutación en la cultura procesalista, que debe superar una serie de actitudes obsoletas, formalistas, legadas por la dogmática tradicional. Esta mutación, ya en marcha aquí y allá en algunas áreas avanzadas de la doctrina procesal, debería seguir tres direcciones recíprocamente complementarias e integradas: la globalización del análisis, fruto indispensable del hecho de que muchos de los problemas que surgen tienen ya dimensiones que no se limitan a ordenamientos particulares, sino que tienden a afectar a nivel mundial a las instituciones judiciales y a las administraciones de justicia; el empleo sistemático de la comparación, como método de análisis y de confrontación de las líneas de evolución de los diversos sistemas procesales y como instrumento para formular y hacer circular informaciones y posibles soluciones en relación con estos problemas; el estudio interdisciplinar de los temas relativos a la justicia y a la ley procesal, siendo ya evidente la necesidad de integrar el estudio técnico-jurídico tradicional con el método del análisis sociológico y económico y con las contribuciones que pueden provenir de otras áreas de la cultura, desde la epistemología hasta el análisis del lenguaje y la psicología.

Una segunda dirección debería ser la de la recuperación y la reformulación de los valores fundamentales y de los principios generales que se consideran válidos para el proceso civil y penal en los ordenamientos avanzados en este momento histórico y para un previsible futuro. Es necesaria una redefinición profunda y orgánica de los sujetos a los que hace referencia la administración de justicia, no siendo suficiente el concepto abstracto de 
«sujeto de derecho» o de «parte», y de los intereses o de las situaciones jurídicas que necesitan encontrar tutela en la ley procesal, no siendo adecuada la referencia al catálogo tradicional de los derechos subjetivos procedente de los códigos. Es necesario, además, un análisis más profundo de los derechos procesales y de las garantías constitucionales del proceso, en un cuadro socio-político en el cual, por un lado, no son suficientes las garantías meramente formales pero, por otro lado, el discurso completo sobre los derechos y las garantías debe ser todavía completado en muchos ordenamientos.

Una tercera dirección es, en alguna medida, más específica, y hace referencia a una necesaria redefinición sistemática de los instrumentos de tutela procesal. Para mostrar sólo algunos ejemplos, se trata de aclarar la función de la tutela cautelar y sumaria respecto de la tutela ordinaria, para establecer hasta qué punto se pueda extender la primera reduciendo el ámbito de la segunda, aunque sea la tutela ordinaria la que ofrece el máximo de garantías. $\mathrm{O}$ bien, se trata de reformular el problema de la actuación ejecutiva de los derechos sobre el plano de la exigencia de efectividad de la tutela, superando las lagunas y las ineficiencias que caracterizan a muchos ordenamientos procesales. Aún más, se trata de establecer cuándo y sobre qué materias resulta irrenunciable el recurso a la «justicia del Estado» y cuándo, en cambio, es razonable dejar espacio a formas alternativas, o bien cuándo es necesario acudir a la justicia «pública» y cuando, en cambio, es más oportuno el recurso a la justicia «privada».

El elenco de las tareas a realizar, así como de los problemas metodológicos y culturales que esperan a ser resueltos, podría continuar todavía más, pero no es ésta la ocasión para escribir un completo «libro de los sueños». Por otra parte, la experiencia muestra que la dialéctica entre racionalidad y crisis de la ley procesal no se sitúa en un momento específico de la historia, sino que tiende a ser una suerte de historia sin fin, en cuanto reflejo de los cambios sociales, económicos y políticos que siempre vuelven a proponer nuevas «necesidades de justicia».

(Trad. de Mercedes Fernández López) 